\title{
Molecular Cloning of Hemoglobin Alpha-chain Gene from Pantholops hodgsonii, a Hypoxic Tolerance Species
}

\author{
Yang Yingzhong', Yunden Droma², Jin Guoen ${ }^{1}$, Bai Zhenzhong1, Ma Lan', \\ Yun Haixia ${ }^{1}$, Cao Yue ${ }^{1}$, Keishi Kubo ${ }^{2}$ and Ge Rili,* \\ ${ }^{1}$ Research Center for High Altitude Medical Sciences, Qinghai University Medical School, Xining 810001, P. R. China \\ ${ }^{2}$ First Department of Internal Medicine, Shinshu University School of Medicine, Matsumoto 390-8621, Japan
}

Received 19 July 2006, Accepted 15 January 2007

To investigate the possible mechanisms of high-altitude native animals in adapting to high altitude, we cloned hemoglobin alpha-chain (alpha-chain $\mathrm{Hb}$ ) gene from Pantholops hodgsonii, an animal species that indigenously lives at elevations of 3700-5500 $\mathrm{m}$ on the Qinghai-Tibetan plateau. Using reverse transcription polymerase chain reaction (RT-PCR) technique, the alpha-chain $\mathrm{Hb}$ gene was amplified from total RNA in the liver of the Pantholops hodgsonii. TA cloning technique was used and the PCR product was cloned into pGEM-T vector. The DNA sequence of the gene was highly homologous with sheep $(99.1 \%)$, goat $(98.6 \%)$, cattle $(95.6 \%)$ and human (86.5\%). The alpha-chain Hb gene encoded a 142-amino acid protein that could be identified with the homology of alpha-chain Hb protein in sheep $(98 \%)$, goat $(96 \%)$, cattle $(91 \%)$ and human $(87 \%)$. However, 18 alternations were detected when compared with the alpha-chain $\mathrm{Hb}$ gene in human, and 2 in sheep. Moreover, the alterations of á117 GluAsp and $\alpha 132$ AsnSer in important regions were noted in human and sheep, respectively. Phylogenetic analysis suggested that the structure of alpha-chain $\mathrm{Hb}$ was highly similar to that in sheep. This study provided essential information for elucidating the possible roles of hemoglobin in adapting to extremely high altitude in Pantholops hodgsonii.

Keywords: Adaptation, Alpha-chain $\mathrm{Hb}$, Molecular cloning, Pantholops hodgsonii, Qinghai-Tibetan plateau

\footnotetext{
*To whom correspondence should be addressed.

Tel: 86-971-6142063; Fax: 86-971-6142063

E-mail: geriligao@hotmail.com, yyz77921@hotmail.com
}

\section{Introduction}

As the most prominent terrestrial highland on the earth, the Qinghai-Tibetan Plateau has great effects on global climate. Its flora and fauna prosper on the plateau and are constantly challenged by the harsh environment of hypoxia, low temperature, high solar radiation, and a lack of biological reproduction. The cold climate and hypoxia are the two most important ecological factors restricting viability of plateau animals. Native animals in the Qinghai-Tibetan Plateau, who have survived over thousands of years on the highland, must have developed their own mechanisms of adaptation to harsh environmental stress during their long evolutional history (Xu et al., 2005).

Pantholops hodgsonii (Tibetan antelope or Chiru) was first described to the Western world by Abel in 1826 and is as an excellent representative of the native mammalian species which have adapted to the Qinghai-Tibetan Plateau. P. hodgsonii belongs to Chordata phylum, Mammalia class, Artiodactyla order, Bovidae family, Pantholops genus. They are mainly distributed over Qinghai Province, Tibetan Autonomous Region and Xinjiang Autonomous Region in China, as well as in India at elevations from 3,700 to 5,500 meters. (Ginsberg et al., 1973; Nowak, 1999; Global Biodiversity Information Facility/Pantholops hodgsonii). They can run for hours at a spectacular speed in this extremely hypoxic environment, and such high speed and stamina indicate that they are effective in oxygen intake, transport and tissue dispersion (Xu et al., 2005).

Hemoglobin $(\mathrm{Hb})$, the key component of oxygen storage and regulation, is widely distributed in all living organism including animals, plants, bacteria, yeast, etc (Hardison, 1998). It is unique in its ability to adapt to a wide range of environmental conditions (Mônic et al., 2003). In order to contribute to the understanding of the genetic background underlying the structure and function of the $\mathrm{Hb}$ in $P$. hodgsonii at high altitude, the cDNA which codes the alpha-chain $\mathrm{Hb}$ has been characterized. 


\section{Materials and Methods}

Sample preparation. The current animal genetic study was approved by the Ethics Committee of Qinghai University (Xining, China) and the China Zoological Society. The protocol of the investigation was in accordance with the principles outlined in the China Practice for the Care and Use of Laboratory Animals.

An adolescent $P$. hodgsonii was captured in Ke-ke-xi-li Natural Reservation (altitude 4,783m) in Qinghai Province, People's Republic of China in December 2004. The animal was sacrificed at the spot. The samples from various organs and tissues were rapidly taken and immediately stored into liquid nitrogen. All of the instruments were treated under 180 for $8 \mathrm{hrs}$, and all of the reagents were dissolved with DEPC treated water.

RNA isolation. Total RNA was extracted and purified from the liver tissue of $P$. hodgsonii using TRIZOL reagent (Invitrogen, Co., Ltd.) following the manufacturer's protocol. The concentrations of RNA samples were quantified with EPPENDORF 6131 nuclear detector (EPPENDORF, Co., Ltd.) and the samples showed a ratio of $\mathrm{A}_{260} / \mathrm{A}_{280}>1.8$ were used for following experiment.

RT and PCR. Reverser transcriptational reaction was carried out as the following: $2.0 \mu \mathrm{g}$ aliquots of the total RNA were mixed with $0.5 \mu \mathrm{g}$ oligo $\mathrm{dT}_{15}$ in $8 \mu \mathrm{l}$ volume and pretreated with $70^{\circ} \mathrm{C}$ for $5 \mathrm{~min}$, then it was transferred under $4^{\circ} \mathrm{C}$ for $2 \mathrm{~min} .5 \times \mathrm{M}$-MLV Buffer, M-MLV Transcriptase (Promega, Co., Ltd.), $2.5 \mathrm{mM}$ dNTP, Rnasin were added into the mixture by the manufacturer's protocol, respectively. The final volume was adjusted by DEPC $\mathrm{ddH}_{2} \mathrm{O}$ up to $20 \mu \mathrm{l}$. The reaction was lasted $45 \mathrm{~min}$ under $37^{\circ} \mathrm{C}$, and then stored at $-20^{\circ} \mathrm{C}$

Our previous study revealed that $P$. hodgsonii is more closely related with $O$. aries, $C$. hircus, and $O$. oreotragus, rather than other antelope species (the Antilopinae subfamily) (Xu et al., 2005). Therefore, the primers applied to the current PCR experiments were designed according to the DNA sequence of sheep (X70214) and bovine (BC102940) alpha-chain $\mathrm{Hb}$ in GenBank. These primers were produced by Invitrogen (Invitrogen, Co., Ltd.), in which the forward primer was HbA-F: 5'-ACCCACCATGGTGCT GTCTG C-3' and the reverse primer was HbA-R: 5'-GGAGGTGAGA GTGCGCAGAGC-3'. The PCR cycles were comprised of predenaturation at $95^{\circ} \mathrm{C}$ for $5 \mathrm{~min}, 30$ cycles of $95^{\circ} \mathrm{C}$ for $30 \mathrm{~s}, 55^{\circ} \mathrm{C}$ for $1 \mathrm{~min}$, and $72^{\circ} \mathrm{C}$ for $1 \mathrm{~min}$, followed by a final elongation at $72^{\circ} \mathrm{C}$ for $10 \mathrm{~min}$. The $501 \mathrm{bp} \mathrm{PCR}$ products were eluted from the agarose gel and purified with QIAquick Gel Extraction Kit (Clontech, Co., Ltd.), and cloned into the pGEM-T vector with a ligation system supplied by Promega.

DNA sequencing. All of the recombinants which were selected by white-blue selection method were sequenced by ABI 377 DNA Analyzer using the dye-terminator chemistry (Invitrogen, Co., Ltd.). The homology sequence search was carried out by an ALIGNMENT program of BIOEDIT. The phylogenetic tree was constructed according to the amino acids sequences of alpha-chain $\mathrm{Hb}$ from $P$. hodgsonii and other species using neighbor-joining methods by the ClustalW program within MEGA version 3.1. The cDNA sequence of alpha-chain $\mathrm{Hb}$ has been submitted to GenBank (Accession number DQ 650713).

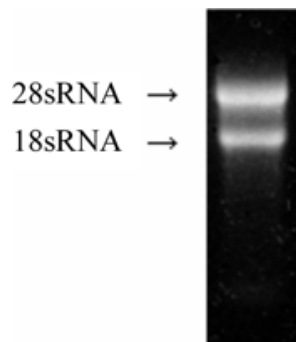

Fig. 1. The total RNA of liver tissue of Tibetan antelope by the formaldehyde agarose gel electrophoresis.

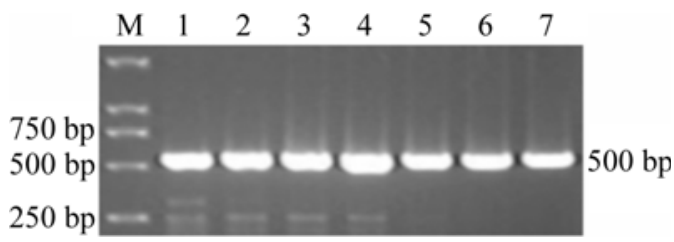

Fig. 2. Gradient PCR product of the cDNA of alpha-chian $\mathrm{Hb}$ of Pantholops hodgsonii by special primers in $1 \%$ agarose gel. (Lane 1-7 shows the results under different annealing temperatures from 54 to $60^{\circ} \mathrm{C}$. There are two unspecific bands near $250 \mathrm{bp}$ in lane 1 , with the annealing temperature increasing; we can see only one band in lanes 2,3 , and 4 . Once the annealing temperature arrived at $58^{\circ} \mathrm{C}$, there were no unspecific bands during the PCR reaction, and the picture shows a specific result in lanes 5, 6, and 7 after the PCR reaction was optimized with a believable result)

\section{Results}

Total RNA extraction. Trizol reagent was used in RNA isolation from the $P$. hodgsonii liver by a one step method. The product was detected on $0.8 \%$ formaldehyde agarose gel electrophoresis (Fig. 1). An Eppendrof spectrophotometer was used to measure the concentration of the total RNA. A ratio of $\mathrm{A}_{260} / \mathrm{A}_{280}>1.9$ sample was used in RT-PCR.

The PCR amplification of alpha-chain $\mathrm{Hb}$ gene. The alpha-chain $\mathrm{Hb}$ from $P$. hodgsonii was amplified using PCR technique. PCR products were detected by $1.5 \%$ agarose electrophoresis, and then a 500 bp DNA segment was seen, which was expected (Fig. 2).

The cloning of target gene and selection of recombinant. The purified PCR product of alpha-chain $\mathrm{Hb}$ was linked to pGEM-T vector, and then the recombinant plasmid was successfully constructed through $\alpha$-complementation method, $\mathrm{T}_{7}-\mathrm{SP}_{6} \mathrm{PCR}$ amplification analysis and sequential analysis.

Characterization of cDNA from $\boldsymbol{P}$. hodgsonii. Since the deduced amino acid sequence was highly homologous to alpha-chain $\mathrm{Hb}$ of other species reported thus far, we concluded this to be the $P$. hodgsonii alpha-chain $\mathrm{Hb}$. We cloned the cDNA sequence of the alpha-chain $\mathrm{Hb}$ in $P$. hodgsonii and therefore predicted the amino acid sequence of 


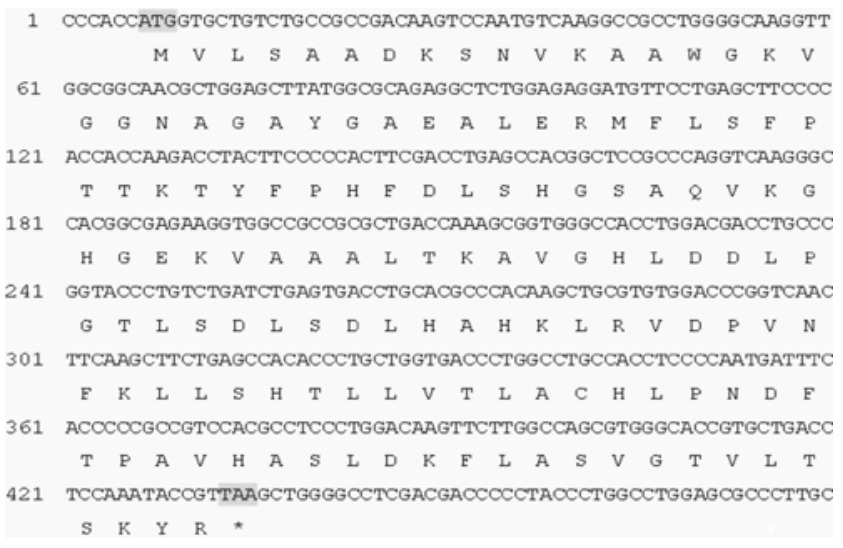

Fig. 3. The nucleotide and deduced amino acid sequence of alpha-chain $\mathrm{Hb}$ of Pantholops hodgsonii. It contains a 429nucleotide coding region. The initiation codon $(\mathrm{M})$, the frame termination codon $(*)$, respectively, are shaded. The sequence is deposited in GenBank in an accession number of DQ650713.

the alpha-chain $\mathrm{Hb}$. This cDNA contained an open reading frame containing $429 \mathrm{bp}$ in size and encodes a protein with 142 amino acids (Fig. 3). The DNA sequence is highly homologous with sheep $(99.1 \%)$, goat $(98.6 \%)$, cattle $(95.6 \%)$, humans $(86.5 \%)$, and the 142 -amino acid protein could be identified as alpha-chain $\mathrm{Hb}$ of sheep (98\%), goat $(96 \%)$, cattle (91\%) and humans (87\%). (Fig. 4, Table 1). The sequence is deposited in GenBank and the accession number is DQ650713.
Mutants in alpha-chain $\mathrm{Hb}$ in $\boldsymbol{P}$. hodgsonii. When comparing the sequence of alpha-chain $\mathrm{Hb}$ of $P$. hodgsonii with that of humans, the $\alpha 117$ Glu $\rightarrow$ Asp alteration can be noted in an important region. This position contacts the two subunits of $\mathrm{Hb}\left(\alpha_{1} \beta_{1}\right.$ interface) which is involved with $\mathrm{Hb}$ oxygenation. Other alterations are: $\alpha 5$ Pro $\rightarrow$ Ala, $\alpha 9 \mathrm{Thr} \rightarrow$ Ser, $\alpha 20$ Al $\alpha \rightarrow$ Gly, $\alpha 21$ His $\rightarrow$ Asn, $\alpha 24$ Glu $\rightarrow$ Al $\alpha, \alpha 61$ Lys $\rightarrow$ Glu, $\alpha 65$ Asp $\rightarrow$ Al $\alpha, \alpha 72$ Al $\alpha \rightarrow$ Gly, $\alpha 74$ V $\alpha$ l $\rightarrow$ Leu, $\alpha 77$ Met $\rightarrow$ Leu, $\alpha 79$ Asn $\rightarrow$ Gly, $\alpha 80$ Al $\alpha \rightarrow$ Thr, $\alpha 83$ Al $\alpha \rightarrow$ Asp, $\alpha 105$ Cys $\rightarrow$ Thr, $\alpha 112$ Al $\alpha \rightarrow$ Cys, $\alpha 116$ Al $\alpha \rightarrow$ Asn, and $\alpha 134$ Ser $\rightarrow$ Gly (Fig. 5). These alternations are in structural positions. However, when compared with the sequence of alpha-chain $\mathrm{Hb}$ of sheep, only two alternations can be observed at position 132 and 134 (Fig. 6).

Phylogenetic analysis. The phylogenetic tree was constructed according to the amino acids sequences of alpha-chain $\mathrm{Hb}$ from $P$. hodgsonii, $O$. aries, $C$. hircus and other species using neighbor-joining methods by the ClustalW program within MEGA version 3.1. Our result indicates that the alpha-chain of $\mathrm{Hb}$ from $P$. hodgsonii is more related to $O$. aries', then $C$. hircus' (Fig. 7).

\section{Discussion}

The $P$. hodgsonii is the unique genus of large endemic mammals adapted to the high elevations and dry climate of the Qinghai-Tibetan Plateau, ranging from $98^{\circ} \mathrm{E}$ westward to

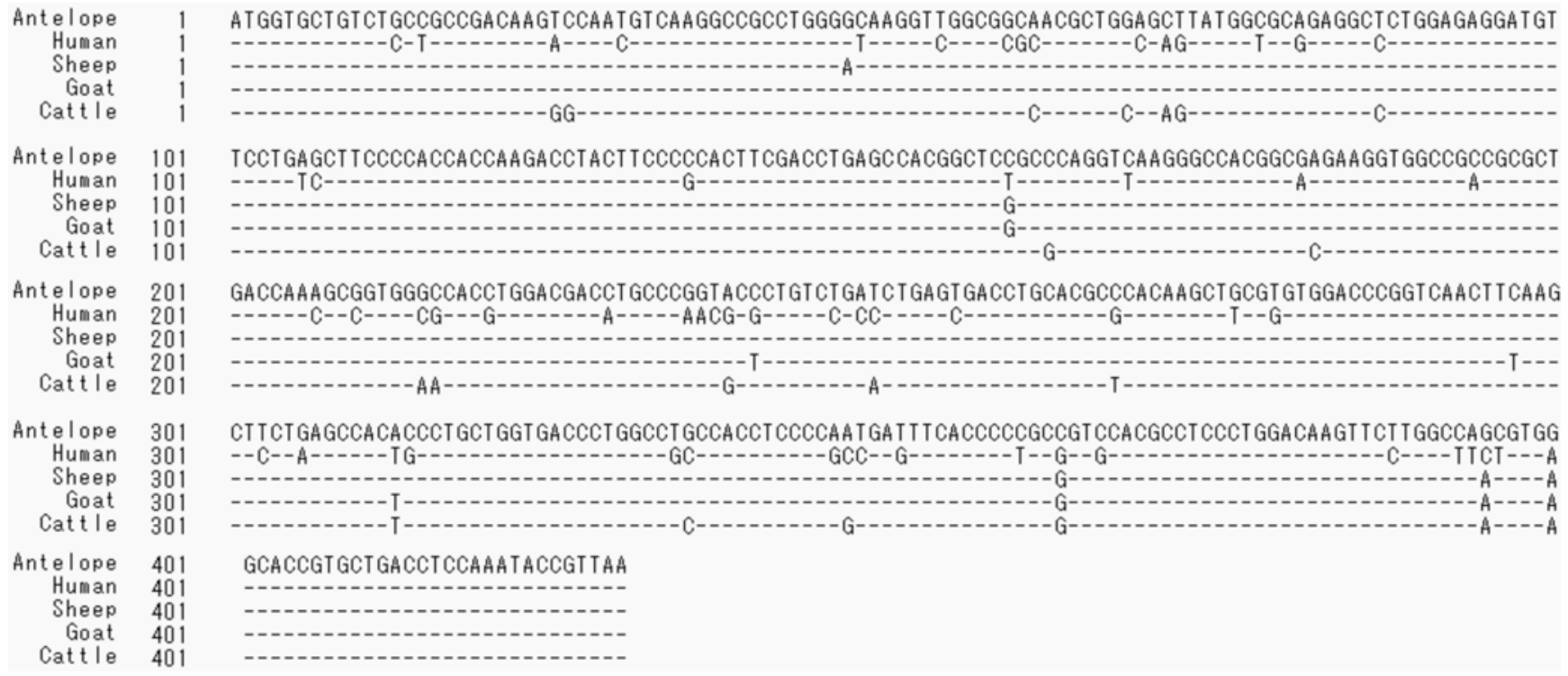

Fig. 4. Multiple CDS sequence alignment of Pantholops hodgsonii alpha-chain $\mathrm{Hb}$ with those of humans (GenBank Accession No.NM_000558), sheep (GenBank Accession No.X70213), goat (GenBank Accession No.J00043) and cattle (GenBank Accession No.BC102940). Numbers with each line indicate the nucleotide sequence number. Areas of nucleotide sequence identity between the antelope, human, sheep, goat and cattle alpha-chain $\mathrm{Hb}$ are indicated by dashes. 
Table 1. Homology comparisons of the nucleotide and amino acids of alpha-chain Hb between Pantholops hodgsonii and other species

\begin{tabular}{ccccc}
\hline The levels of comparison & Human & Sheep & Goat & Cattle \\
\hline The sequence of open reading frame & $86.5 \%$ & $99.1 \%$ & $98.6 \%$ & $95.6 \%$ \\
Amino Acids & $87 \%$ & $98 \%$ & $96 \%$ & $91 \%$ \\
\hline
\end{tabular}

Ladakh in India. The sexes segregate almost completely during the spring and early summer (May and June), when adult females and their female offspring migrate north to certain calving grounds and return south by late July or early August, covering distances as long as $300 \mathrm{~km}$ each way. Seasonal migrations by $P$. hodgsonii constitute a critical aspect of the species' life cycle and help to define the ecosystem as a whole. Domestic yaks and Tibetan sheep, root voles (Microtus oeconomus), upland buzzard (Buteo hemilasius), steppe polecats (Mustela eversmanni), and weasels (Mustela altaica) are also the main vertebrates in the meadow ecosystem. (Ginsberg et al., 1973; Global Biodiversity Information Facility/Pantholops hodgsonii)

Hemoglobin, the key component of oxygen storage and regulation system, is widely distributed in all living organism including animals, plants, bacteria, yeast, etc (Hardison, 1998). Hypoxia affects oxygen transport properties of hemoglobin and alters oxygen affinity by several mechanisms. All modifications adopted by animals appear to optimize both arterial oxygen loading and peripheral unloading (Mairbaurl, 1994). The hemoglobin affinity for oxygen allows rapid adjustments of oxygen binding and release since the process is far less energy demanding than an increase in cardiac output. This adaptation has been attributed to changes in the primary structure of globin chains, which modulates oxygen uptake and delivery to the tissues. Among vertebrates, birds occupy a unique position in terms of their ability to maintain an efficient oxygen supply to the brain during severe hypoxia, which an important adaptation is contributing to their exceptional tolerance at extreme altitudes. It was clarified that

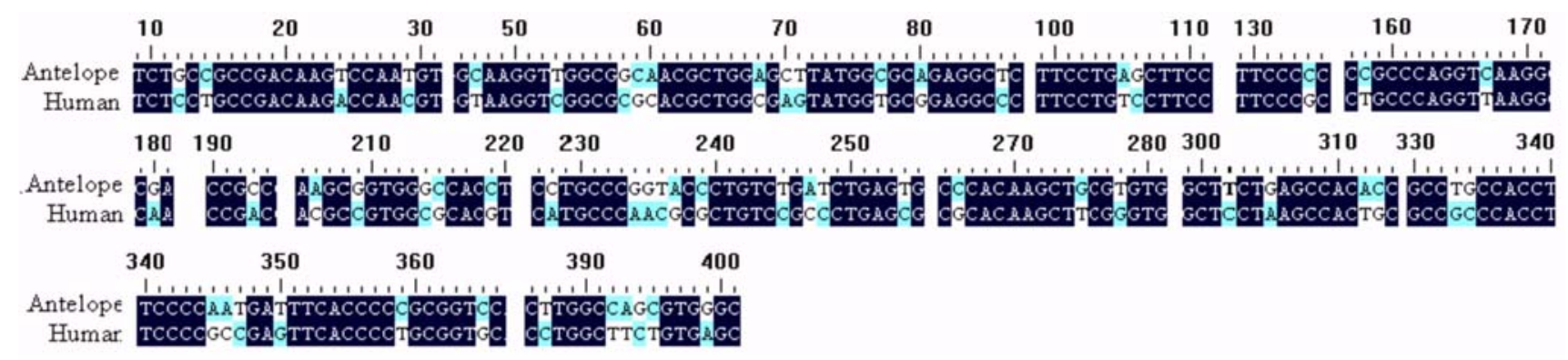

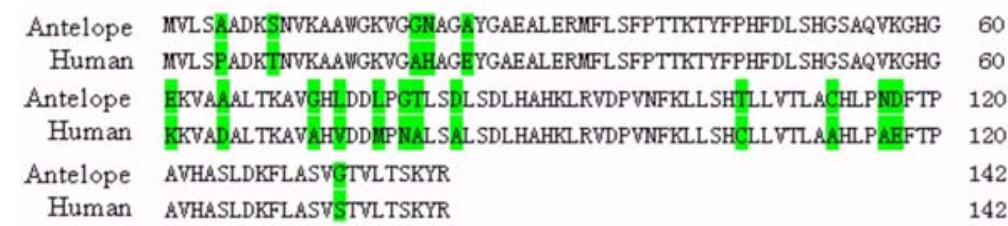

Fig. 5. The comparison of the nucleotide sequence and amino acids sequence of alpha-chain Hb between Pantholops hodgsonii and Humans (GenBank Accession No.NM_000558).

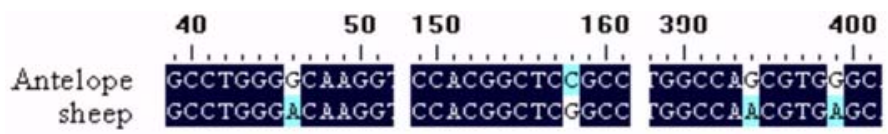

$\begin{array}{rlr}\text { Antelope } & \text { MVLSAADKSNVKAAWGKGGNAGAYGAEALERMFLSFPTTKTYFPHFDLSHGSAQVKGHG } & 60 \\ \text { sheep } & \text { MVLSAADKSNVKASWGKGGNAGAYGAEALERMFLSFPTTKTYFPHFDLSHGSAQVKGHG } & 60 \\ \text { Antelope } & \text { EKVAAALTKAVGLDDLPGTLSDLSDLHAHKLRVDPVNFKLLSHTLLVTLACHLPNDFTP } & 120 \\ \text { sheep } & \text { EKVAAALTKAVGHLDDLPGTLSDLSDLHAHKLRVDPVNFKLLSHTLLVTLACHLPNDFTP } & 120 \\ \text { Antelope } & \text { AVHASLDKFLASVGTVLTSKY } & 142 \\ \text { sheep } & \text { AVHASLDKFLANVSTVLTSKYR } & 142\end{array}$

Fig. 6. The comparison of the nucleotide sequence and amino acids sequence of alpha-chain Hb between Pantholops hodgsonii and sheep (GenBank Accession No. X70213). 


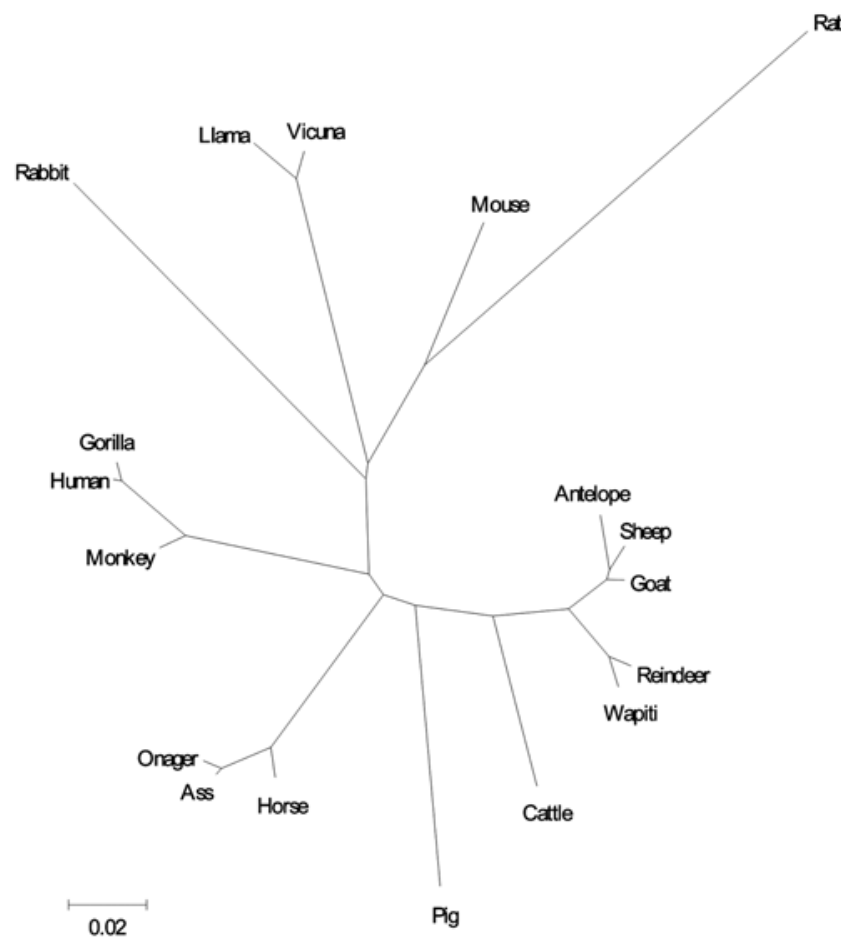

Fig. 7. Phylogenetic tree showing the relationships among the alpha-chain $\mathrm{Hb}$ of Pantholops hodgsonii with those of other animals. The amino acid sequence used for the analysis were obtained from the National Center for Biotechnology Information protein database with the following accession numbers: Human (CAA23752), Sheep (CAA49750), Goat (AAA30909), Cattle (AAI02941), Horse (P01958), Pig (P01965), Rabbit (CAA24244), Rat (NP_037228), Mouse (NP_032244), Cat (P07405), Llama (P01973), Vicuna (P07425), Gorilla (P01923), Reindeer (P21379), Ass (P01959), Wapiti (AAY57619), Onager (AAB93465). The sequences were aligned by ClustalW program and the phylogenetic tree was constructed by neighbor-joining methods within MEGA version 3.1 .

mutants in birds $\mathrm{Hb}$ gene raises the oxygen affinity. The barheaded goose lives on Tibetan lakes and migrates across the Himalayas to India during the winter. Another species, the Andean goose, lives in the High Andes all year round. Possession of an $\mathrm{Hb}$ with high oxygen affinity helps to adapt bar-headed and Andean geese to high altitudes. The $\mathrm{Hb}$ amino acid residues $\alpha-119$ and $\beta-55$, which form a $\alpha_{1} \beta_{1}$ contact in human hemoglobin, are altered in bar-headed and Andean geese, respectively and suggest that loss of this contact increases $\mathrm{O}_{2}$ affinity (Hiebl et al., 1987; Godovac-Zimmermann et al., 1988; Jessen et al., 1991; Weber et al., 1993; Petruzzelli et al., 1996). Others, llama, Guanaco and alpaca show a comparable high blood oxygen affinity it adapted to high altitude so smoothly, also caused by the substitution $\beta 2$ (NA2) HisAsn (Marco et al., 1990); the substitution of valine at position 135 in the $\beta^{\Pi}$-chain may be responsible for the high intrinsic oxygen affinity of yak hemoglobin (Lalthantluanga et al., 1985).

In this study, nucleotide sequence analysis of $P$. hodgsonii alpha-chain $\mathrm{Hb}$ mRNA was considerably expedited by the availability of plasmids that contained an insert of a cDNA copy of the alpha-chain $\mathrm{Hb}$ mRNA. The cDNA contained a 429 bp open reading frame encoding a peptide of 142 amino acids which was identified by PCR technique. The key point is that our previous work on phylogenetic analysis on cytochrome b genes revealed that $P$. hodgsonii is more closely related with $O$. aries, $C$. hircus, and $O$. oreotragus, rather than other antelope species (the Antilopinae subfamily). The proper primers we designed were the main reason for successful cloning. The nucleotide sequences of the coding regions of $P$. hodgsonii and sheep alpha-chain $\mathrm{Hb}$ mRNAs share a great deal of homology where the deduced protein sequences differ only by 132 and 134 of 142 amino acids. Compared with human alpha-chain $\mathrm{Hb}$, there are 18 differences. Regarding structural alterations, $\alpha 117$ GluAsp is particularly important. It is located at $\alpha_{1} \beta_{1}$ contact which is known to be very tight and remains largely unchanged during the quaternary conformational change that accompanies ligand binding at the heme site in normal human HbA (Petruzzelli et al., 1996). And differences especially 132 may also play an important role in $P$. hodgsonii oxygen transportation at high-altitude when compared with sheep alpha-chain $\mathrm{Hb}$. We are now cloning beta-globin of $P$. hodgsonii and detecting mutations which possibly explain the mechanism in $P$. hodgsonii adaptation to high-altitude.

In conclusion, this study determined the amino acid sequence of alpha-chain $\mathrm{Hb}$ of the $P$. hodgsonii, allowing the comparisons of the amino acid sequence between $P$. hodgsoni and humans, and other vertebrates, thereby, characterizing the intrinsic properties of $\mathrm{Hb}$.

Acknowledgments We are grateful to Prof. Yu Mingsheng of the Qinghai Academy of Animal and Veterinary Science, Qinghai University, and staff of the Ke-ke-xi-li Natural Reservation. This work was supported by "National Basic Research Program of China, No.2006CB504100" and National Natural Science Foundation of China, No. 30393133.

\section{References}

Ginsberg, J. R., Schaller, G. B. and Lowe, J. A. Petition to List the Tibetan Antelope (Pantholops hodgsonii) as an Endangered Species Pursuant to the U. S. Endangered Species Act of 1973. URL:http://www. earthisland.org/tpp/Exec_Summ.htm

Global Biodiversity Information Facility/Pantholops hodgsonii. URL:http://www.secretariat.gbif.net/portal/ecat_browser.jsp?taxon Key $=1009055 \&$ country Key $=0 \&$ resource Key $=0$.

Godovac-Zimmermann, J., Kosters, J., Braunitzer, G and Goltenboth, R. (1988) Structural adaptation of bird hemoglobins to highaltitude respiration and the primary sequences of black-headed gull (Larus ridibundus, Charadriiformes) alpha A- and beta/ beta'-chains. Biol. Chem. Hoppe. Seyler. 369, 341-348.

Hardison, R. (1998) Hemoglobins from bacteria to man: evolution of different patterns of gene expression. J. Exp. Biol. 201, 
1099-1117.

Hiebl, I., Braunitzer, G. and Schneeganss, D. (1987) The primary structures of the major and minor hemoglobin-components of adult Andean goose (Chloephaga melanoptera, Anatidae): the mutation Leu----Ser in position 55 of the beta-chains. Biol. Chem. Hoppe. Seyler. 368, 1559-1569.

Jessen, T. H., Weber, R. E., Fermi, G., Tame, J. and Braunitzer, G. (1991) Adaptation of bird hemoglobins to high altitudes: demonstration of molecular mechanism by protein engineering. Proc. Natl. Acad. Sci. USA 88, 6519-6522.

Lalthantluanga, R., Wiesner, H. and Braunitzer, G. (1985) Studies on yak hemoglobin (Bos grunniens, Bovidae): structural basis for high intrinsic oxygen affinity? Biol. Chem. Hoppe. Seyler. 366, 63-68.

Mairbaurl, H. (1994) Red blood cell function in hypoxia at altitude exercise. Int. J. Sports. Med. 15, 51-63.

Mônica, B. M., Silvana, B., Adriana, S. S., Duarte, S. H. O, Márcio, A., Torsoni, S. T. O. S. and Fernando, F. C. (2003) Molecular characterization of hemoglobin $\alpha-\mathrm{D}$ chains from Geochelone carbonaria and Geochelone denticulata land turtles. Comp. Biochem. Physiol. B: Biochem. Mol. Biol. 134, 389-395.
Nowak, R. M. (ed.) (1999) Walkers Mammals of the World, Sixth Edition. The Johns Hopkins University Press, Baltimore, USA.

Petruzzelli, R., Aureli, G., Lania, A., Galtieri, A., Desideri, A. and Giardina, B. (1996) Diving behaviour and haemoglobin function: the primary structure of the $\alpha$ - and $\beta$-chains of the sea turtle (Caretta caretta) and its functional implications. $J$. Biochem. 316, 959-965.

Piccinini, M., Kleinschmidt, T., Klaus, D. J. and Braunitzer, G. (1990) Primary structure and oxygen-binding properties of the hemoglobin from guanaco (Lama Guanacoe, Tylopoda). Bio. Chem. Hoppe. Seyler. 371, 641-648.

Weber, R. E., Jessen, T. H., Malte, H. and Tame, J. (1993) Mutant hemoglobins (alpha 119-Ala and beta 55-Ser): functions related to high-altitude respiration in geese. J. Appl. Physiol. 75, 26462655.

Xu, S. Q., Yang, Y. Z., Zhou, J., Jing, G. E., Chen, Y. T., Wang, J., Yang, H. M., Wang, J., Yu, J., Zheng, X. G. and Ge, R. L. (2005) A mitochondrial genome sequence of the tibetan antelope (Pantholops hodgsonii). Genomics Proteomics Bioinformatics 3, 5-17. 